\title{
El sushi en Japón y en el mundo ${ }^{1}$
}

DOI: $10.32870 /$ mycp.v11i32.357

Daisuke Kishi ${ }^{2}$

Resumen

El sushi es quizá uno de los platillos tradicionales de Japón más conocidos internacionalmente. En este artículo se intenta señalar en forma breve cuál es la autenticidad de los sushis, a partir de su origen y variedad. Asimismo se explica la globalización del mencionado alimento, la cual se debe en mayor o menor grado al paladar de la gente, costumbres e ingredientes que se consiguen en su tierra.

Abstract

The sushi is maybe one of the most famous Japanese traditional dishes worldwide. This article tries to briefly point out which is the authenticity of the sushis, from its origin and variety. It also explains the globalization of the mentioned food; this globalization is due to the palate of people, habits and ingredients that they obtain on their land.

\section{Introducción}

La comida japonesa indudablemente es uno de los alimentos que están de moda en muchos países del mundo. Si uno viaja al extranjero, sea el país que sea, se encontrarán, al menos en las ciudades, grandes restaurantes japoneses donde seguramente tienen una variedad de sushi. En México también los hay muchos, quizá cada día más, pero una característica que distingue a otros países probablemente es la existencia de puestos de comida japonesa u oriental. Entre ellos destacan los de sushi. Desde el punto de vista cultural o social

1. Este artículo está basado en una conferencia sobre "el sushi - ¿una moda alimenticia o una globalización cultural?”, la cual di el día 13 de octubre de 2005 en el Encuentro Internacional de Vinculación Empresa-Escuela, Asia-Jalisco.

2. Profesor-investigador del Departamento de Estudios del Pacífico de la Universidad de Guadalajara. 
esto puede deberse a una influencia de los puestos de tacos o de fritangas. Entonces, ¿por qué está tan de moda comer sushis? ¿Es porque se considera un alimento dietético y saludable, o simplemente porque está rico? Según Kodansha Encyclopedia of Japan (1983), el mencionado alimento japonés se clasifica en cuatro categorías: a) maki-zushi; b) niguiri-zushi; c) chirashi-zushi, y 4) hako-zushi, las cuales mencionaremos posteriormente.

El sushi es un platillo tradicional de Japón que se prepara básicamente con arroz mezclado con un poco de vinagre ligeramente dulce y pescado crudo fresco. Un japonés o un conocedor de Japón, al observar bien el sushi que se vende en México y tal vez en la mayor parte del mundo, encontrará la diferencia que existe entre el sushi auténtico y el extranjero. Diríamos nosotros que es un pseudosushi o un cuasi sushi. Posteriormente analizaremos el porqué.

\section{Origen del sushi}

Etimológicamente la palabra sushi, que se escribe en japonés 鮨 o 寿司 tiene el siguiente significado: en el primer kanji, la parte izquierda tiene un valor de "pez o pescado", y la derecha significa "madurar algo" (en este caso, al pescado). La última palabra formada por dos kanjis: 寿(“felicidad”) y 司 (“gobernar”), adaptados a la forma de ser de los japoneses, significa "gobernar la felicidad", ya que en general muchos japoneses hemos sido supersticiosos y tendemos a relacionar lo que nos está pasando con el bien o con el mal. Es importante mencionar también que desde el punto de vista fonético los nombres maki-sushi y niguirisushi - que suelen encontrarse en el menú del restaurante japonés en el extranjero- no son correctos, sino que se deben pronunciar maki-zushi y niguirizushi, respectivamente. Este fenómeno, común en muchos

De hecho el sushi, sorprendentemente, no es originario de Japón; su origen, según algunos investigadores, parece estar en el sureste de Asia y data del siglo IV a. C. idiomas, se debe a la sonorización de [s] entre vocales.

De hecho el sushi, sorprendentemente, no es originario de Japón; su origen, según algunos investigadores, parece estar en el sureste de Asia y data 
del siglo IV a. C. ${ }^{3}$ En esa época se conservaba pescado, en especial de agua dulce dentro del arroz u otro tipo de cereal cocido que se iba fermentando, pero ese arroz fermentado no se comía. Parece que esta técnica, a través de China y posteriormente de Corea, llegó a Japón en la Era de Nara. ${ }^{4}$ Con razón, entre la comida coreana y la japonesa se encuentran platillos comunes o muy similares; desde luego que el caso del sushi no es excepción. Según el Código Yoro llamado Yoro-ritsuryo (718), se pagaban los impuestos con sushi de abulón (Morieda, 2002: 56). En ese periodo nipón se comía el supuesto sushi arriba mencionado también con tenca o una especie de trucha. Es evidente que durante los siglos X al XII dicho alimento todavía se consumía de la misma manera que en las primeras etapas, según se afirma en algunas obras literarias japonesas. ${ }^{5}$

En la Era de Azuchi-Momoyama, ${ }^{6}$ pocos años antes de la Era de Edo, ${ }^{7}$ a finales del siglo XVI, empezó a usarse el vinagre, gracias al cual el sushi tuvo un desarrollo fabuloso, ya que el vinagre impide que los alimentos se echen a perder; se supone que ahí empezó a consumirse el pescado acompañado de arroz como si fuera un solo platillo. En otras palabras, podemos decir que es el verdadero origen del sushi actual. Se empezaron a consumir pescados de agua salada tales como huachinango pequeño o caballa, entre otros. A finales del periodo de Edo (probablemente entre 1804 y 1830), el niguiri-zushi fue inventado por Yohei Hanaya. Este platillo también recibe el nombre de edomae-zushi (que significa "sushi de la frente de Edo"), puesto que se usaban los pescados del golfo de Tokio, el cual se encuentra enfrente de la capital Edo. De hecho, durante el periodo de Edo y el de Meiji (1868-1912) prácticamente no había restaurantes de sushi, sino puestos de sushi ${ }^{8}$ donde el pescado crudo solía conservarse en vinagre, en salsa de soya o incluso ya preparado. De esta manera, se deduce que la base culinaria del sushi actual se ha formado antes del periodo de Meiji. Después de la Segunda Guerra Mundial, ha cambiado la forma de venta, dado que por cuestión de la higiene se prohibió

3. Véase www.sushihome.cl/historia.htm.

4. Años 710-793. La capital se llamaba en esa época "Heijookyoo".

5. Se puede observar en el Diario de Tosa (Tosa Nikki) en 935; el Libro de la Almohada (Makura no Soshi) en 1002; Antología de los Cuentos del Pasado (Konjaku Monogatari) en 1108 o poco después.

6. Años 1573-1602.

7. Años 1603-1867.

8. Esa escena se puede observar en una obra del pintor Hiroshige Utagawa, la cual se conserva en el Museo Conmemorativo de Artes de Ohta. De la misma manera, se puede acudir a las páginas Web: http://digitalmuseum.rekibun.or.jp/app/collection/detail?id=0190203535\&sr =\%96\%BC\%8F\%8A; http://www.kabuki-bito.jp/special/mec/03/no2.html. 
vender sushis en los puestos. Esa época en la historia del sushi se podría considerar, pues, como una etapa de transición de lo popular a lo lujoso.

\section{Variedad del sushi tradicional}

En este apartado nos referimos a la variedad en un sentido clásico o tradicional, puesto que recientemente existe una variedad más amplia de dicho plato, incluso en la tierra del sol naciente.

\section{Maki-zushi}

Existen inmensas variedades de sushi, dentro de las cuales una de las más conocidas internacionalmente quizá es el llamado maki-zushi, que quiere decir "sushi enrollado". Es muy fácil de preparar no sólo en restaurantes, sino también en casa. Como es sabido por los aficionados a la comida japonesa y muchos otros, en una tablita de madera o de bambú se pone primero una hojita de algas marinas secas llamada nori, ${ }^{9}$ a la cual se le pone de manera uniforme una capa de arroz cocido, pero no en mucha cantidad; luego se le ponen, dependiendo del gusto, pescado crudo o preparado, verduras como pepino u otros ingredientes. Después de este procedimiento se enrolla cuidadosamente la tablita, apretándola al mismo tiempo. Una vez enrollado el sushi, normalmente lo cortamos en seis u ocho pedazos. ${ }^{10}$ Se come con la mano o con los palitos con un poco de salsa de soya, pero no totalmente bañado en ella. Esto para conservar el sabor original del sushi o ingredientes.

El maki-zushi probablemente es el sushi más consumido y arraigado en Japón. Primero, es fácil de comer; en segundo lugar es fácil para llevarse, pues en cualquier supermercado o en la sección de comidas preparadas de un centro comercial, y por supuesto a la entrada de una sushiería o un restaurante de sushi, se puede encontrarlo en un contenedor. A la hora de comida, en Japón a las doce mucha gente lo compra para disfrutarlo en su oficina ya que tienen un tiempo limitado para comer. Para llevarse a un día de campo (ir a día de campo es mucho menos común que aquí) uno acostumbra preparar otro tipo

9. Una hoja nori mide normalmente $20 \times 18 \mathrm{~cm}$. El nori más conocido se llama asakusa-nori, ya que en el periodo de Edo, en la zona de Asakusa, en Tokio se cosechaban dichas algas.

10. Véase http://www.sushi-nori.co.jp/maki.html. 
de lonche, quizá sándwich u oniguiri. ${ }^{11}$ Es interesante añadir que el sushi no es la comida de todos los días, sino que tal vez haya similitud con los antojitos mexicanos.

\section{Niguiri-zushi}

El niguiri-zushi $i^{12}$ es un "sushi apretado", ya que encima de un puño de arroz apretado se pone algún ingrediente, sea un pedazo de atún fresco, calamar, pulpo, huachinango, lenguado, abulón, anguila asada con salsa de soya agridulce, huevo, etc. Y ¿cómo se puede comer más rico? La mejor manera de saborearlo es comerlo en cuanto el cocinero lo prepara. Entonces, ¿de qué manera se come? Por supuesto se puede comer con los palitos, pero yo les recomendaría probarlo con la mano, pues sinceramente es más fácil de tomarlo y comerlo que con los palitos. Además es más conveniente ponerlo en salsa de soya por la parte del ingrediente, o sea la parte del pescado, porque si se intenta al revés se desbarata el arroz. Y la cantidad de salsa de soya deseable es la mínima para que no se pierda el sabor del ingrediente. También es importante mencionar que el wasabi, hecho de una especie de raíz verde rallada, suele acompañar al sushi no sólo por sabor, sino por cuestión de salud, ya que ese picante japonés sirve para desinfectar los alimentos.

Este sushi puede ser uno de los más elegantes, pues aunque varíe mucho el precio, si uno va a una sushiería de primera categoría un par de niguiri-zushi costará 1,000 a 2,000 yenes (100 a 200 pesos). Tal vez por esta razón, cuando tenemos visita o invitados en casa pedimos, con cierta frecuencia, un servicio a domicilio a un restaurante de sushi. Otro ejemplo de interés será el caso de un funeral o de un velorio; al final del rezo en el velorio o después del funeral se invita normalmente un niguiri-zushi, tal vez porque se considera elegante, o sea porque el arroz en la terminología de sushi se llama shari, el cual coincide con el nombre de "hueso del Buda" (Busshari).13

\section{Chirashi-zushi}

11. Es una bola apretada de arroz normalmente en forma triangular, la cual contiene en el centro pedacitos de umeboshi (una especie de chamoy) o salmón salado asado, entre otros ingredientes.

12. Véase http://www.tabiguru.com/img_taberu/01sushi/tanifugi/tanifugi02.jpg.

13. Según la creencia budista, después de la muerte uno va a ser un discípulo del Buda en su mundo. 
El chirashi-zush ${ }^{14}$ quiere decir en japonés "sushi espolvoreado". Sobre el arroz para sushi se esparcen varios ingredientes ya preparados, tales como camarones cocidos, setas, chícharos con toda la cáscara, pepinos, huevos al estilo japonés, etc., la mayoría de los cuales deben ir picados muy finamente. Por eso se ve "espolvoreado", aunque esa expresión parece un poco exagerada. El chirashi-zushi también se llama gomoku-zushi, que significa "sushi de cinco elementos". Este nombre se debe seguramente a lo colorido de los ingredientes. Este sushi quizá sea una de las variedades más caseras, ya que en las reuniones familiares se suele preparar con mucho cariño, lo cual confirman los datos de la encuesta sobre "qué tipo de sushi se prepara en casa". ${ }^{15}$

Tabla 1

\begin{tabular}{llc}
\hline & Tipo de sushi & Frecuencia (\%) \\
\hline 1 & Chirashi-zushi (con los ingredientes & 74.0 \\
& mezclados con el arroz) & \\
2 & Temaki-zushi $^{1}$ & 51.1 \\
3 & Inari-zushi $^{2}$ & 37.7 \\
4 & Chirashi-zushi (con los ingredientes & 24.2 \\
& encima del arroz) & \\
5 & Maki-zushi & 21.2 \\
6 & Oshi-zushi & 5.6 \\
\hline
\end{tabular}

1. Es una variación de maki-zushi. Como indica el nombre te (que significa "las manos"), cada quien lo prepara con sus manos según su gusto, sin usar la tablita. El tamaño de nori es más pequeño y se enrolla en forma de cono.

2. El "inarizushi se hace rellenando bolsas de abura-age endulzado con arroz de sushi" (Hosking, 2001: 246).

Fuente: Mitsukan, 2000.

\section{Hako-zushi}

Según el diccionario japonés Koojien (299 y 1779), el hako-zushi ("sushi en la cajita") se usa como sinónimo de oshi-zushi ("sushi a presión"), ${ }^{16}$ puesto

14. Véase http://shinshu.fm/MHz/50.60/images/qK4p6dSe.jpg; http://party-bambino.com/piclabo/chirashi_11.jpg.

15. Mitsukan, una empresa de vinagre, realizó esta encuesta en el año 2000 tanto en Tokio como en Osaka (Morieda: 136-138).

16. Véase http://www.kouzushi.com/img/osizusi009.jpg en la cual aparece el típico battera; http:// www.sushi.ne.jp/sushinet2/s016merumaga/mz-vol-14.htm en que se explica su preparación. 
que se prepara con arroz envinagrado en una caja de madera que mide unos 13 centímetros cuadrados, encima del cual se colocan pescado en vinagreta, mariscos y a veces verduras. Después con una tapita se presionan todos los ingredientes, para sacarlo del mencionado molde y cortarlo en tamaño de un bocado. Este sushi se considera típico de Osaka, aunque en otras prefecturas tales como Kioto, Toyama, Tottori, Hiroshima y Yamaguchi se producen variantes del mismo. Los pescados más usuales son arenque y caballa.

El mencionado sushi recibe también el nombre de battera, ${ }^{17}$ el cual proviene del vocablo portugués bateira que significa "lancha", ${ }^{18}$ pues en la Era de Meiji, alrededor del 1893 en una sushiería de Osaka bautizaron con ese nombre el sushi hecho con una especie de arenque llamado konoshiro por su forma. Posteriormente se dio dicho nombre al mismo tipo de sushi con caballa en vinagreta.

\section{La corriente actual del sushi en Japón y en el mundo}

En estos días, además de los mencionados sushis clásicos se vende temakizushi, que es seguramente una variación del maki-zushi. Como indica el nombre en sí, se prepara con las manos sin usar esa tablita de madera. De ahí viene la palabra, ya que el prefijo o partícula te significa "manos". Gracias a esta facilidad, parece que se está haciendo cada vez más popular en nuestra vida alimenticia cotidiana. Últimamente cuando realizamos alguna reunión familiar, pero bastante familiar, a veces acudimos a este platillo. Quizá podamos decir que son una clase de "taquitos japoneses". Además de ser fácil de preparar, es divertido, pues cada quien puede servirse a su gusto. Estos elementos nos permiten vivir una vida social un poco más activa, aunque no tanto como en este país.

El sushi, de cierto modo, era antes un platillo muy elegante, pues por ser caro el acceso a los bares o restaurantes de dicha comida estaba limitado a algunas clases de personas. En la actualidad, sin embargo, uno ya puede comerlo a gusto con unos 600 ó 700 yenes en muchos lugares. ¿Dónde se podrá disfrutarlo tan barato? Si encontraste en las calles niponas un letrero que dice: "Kaiten-Zushi", ${ }^{19}$ ya la hiciste. Se refiere al "sushi de banda sin fin".

17. Véase Kojien (1801).

18. De hecho, en la Era de Edo ya existía el portuguesismo battera o batteira, pero sólo en sentido de "lancha" (http://gogen-allguide.com/ha/bateira.html).

19. Véase http://ja.wikipedia.org/wiki/\%E7\%94\%BB\%E5\%83\%8F:KuruKuruSushiRestaurant. jpg\#file. 
Los cocineros lo hacen más mecánicamente; como la banda está girando sin cesar, resulta que los clientes lo comen más rápido y llega gente sin cesar también. Este ritmo hizo posible que el sushi se economizara. Por tal razón, se puede observar que los fines de semana o los domingos, con cierta frecuencia toda la familia acude a estos restaurantes de sushi de banda sin fin, pues últimamente se encuentran sushi de hamburguesa o el de pollo con salsa teriyaki e incluso, como postre, aparecen pasteles o flanes. Esto puede ser una innovación para la tradición japonesa. En este sentido, el sushi se convirtió en una comida más popular y familiar a todas las clases sociales y a través de todas las generaciones. Es importante mencionar también que este sistema se inventó en Osaka en 1958, hace casi medio siglo. La bebida tradicional que acompaña al sushi es el té verde, por supuesto.

En otros países, ¿cuál será la tendencia de dicho alimento? En Corea, por ejemplo, dentro de la categoría de niguiri-zushi existe sushi de carne asada coreana, la cual se prepara generalmente con salsa de soya, ajo, chile y un poco de azúcar. No debemos dejar de mencionar el sushi de kimuchi o kimchi. ${ }^{20}$ De hecho, kimuchi es una combinación de verduras fermentadas tales como hakusai (col china), nabo y puerro junto con camarones fermentados, ajo, chile, ajonjolí, jengibre, sal y azúcar. Este tipo de sushis se podrá considerar como una variación de niguiri-zushi adaptada a la costumbre culinaria de Corea.

En Hong Kong el sushi se puso de moda a partir de los últimos años de los ochenta, en especial debido a la introducción del sistema kaiten-zushi, según lo menciona Wai-ming (301). En esa tierra asiática Genki Sushi y Genroku Sushi son las principales tiendas de cadena en relación con el kaiten-zushi. La primera cuenta en Hong Kong con 18 sucursales, y la segunda con 21. De acuerdo con el mismo autor (302), Genroku Sushi es "el rey de los sushis al estilo hongkonés", dado que usan ingredientes locales, además de que es mayor el tamaño del sushi y más bajo el precio. Esta moda llegó también a Europa; en Londres inauguraron en 1999 Yo! Sushi, y actualmente dicho restaurante de cadenas cuenta con más de 20 sucursales en la misma ciudad.

En algunos países occidentales y americanos, incluyendo Latinoamérica, el llamado California-maki, cuyos ingredientes distintivos son el aguacate y el

20. "La receta más extendida utiliza como ingrediente básico un vegetal conocido como col china (en coreano "Bae-chu"), cuya forma es similar a la de una lechuga, aunque existen otras recetas en las que se utilizan como ingrediente básico rábanos o pepinos. Otros ingredientes son: pimienta roja molida, ajos o cebollas tiernas. De olor fuerte y característico, su sabor es salado y picante." (http://es.wikipedia.org/wiki/Kimchi). 
queso Philadelphia, es ya famosísimo, pero en Japón no existe esta variación. El origen de este sushi, como lo indica su nombre, está en un barrio japonés Little Tokio, en Los Ángeles. En 1963 un cocinero de sushi, Ichiro Mashita, lo inventó en el sushi-bar de Tokio Kaikan. ${ }^{21}$ Dicho invento fue realizado para sustituir toro, que es parte grasosa del atún, por aguacate, ${ }^{22}$ ya que la textura y el sabor con salsa de soya y wasabi del aguacate es similar a los del atún toro.

Podremos decir lo mismo con el sushi de surimi y sushi empanizado. En París incluso existe el sushi de hígado graso (foie gras) del ganso, el cual no se come con palitos sino con cuchillo y tenedor. Estas variaciones pueden ser un invento de los cocineros en el extranjero o un resultado de la adaptación al estilo occidental.

Por último, desde el punto de vista médico o nutritivo, el sushi puede ser un alimento muy sano. En primer lugar, el vinagre que contiene el arroz de sushi permite que se conserve en buen estado el alimento; además previene cansancio físico y presión alta arterial. En segundo lugar, comer pescados como atún (en especial la parte de la panza), caballas, sardinas puede prevenir cáncer, demencia senil, artritis reumatoide, etc. Este efecto se debe al funcionamiento del DHA (ácido docosahexaenoico). Además, las algas marinas son buenísimas para mejorar el funcionamiento de los intestinos. Así que no hay que desperdiciar estos recursos marinos.

\section{Conclusión}

El sushi, por lo visto, parece estar de moda tanto en Japón como en muchos países del mundo, pero no simplemente porque es un alimento extranjero sino por su riqueza nutritiva y tal vez también por su efecto dietético, ejercido en especial por el vinagre. Socialmente —en el caso de Japón-creemos que el sushi desempeña un papel importante para mantener unida una familia y romper el hielo entre la gente.

En el mundo el consumo de sushi seguramente va aumentando cada día más, gracias a su riqueza en nutrición y sabor. En algunos países asiáticos y europeos la introducción del sistema kaiten-zushi en el mercado tal vez sea un inicio del boom de sushi. Las variaciones del sushi en los diversos países no

21. Véase http://ja.wikipedia.org/wiki/Sushi.

22. Véase http://www.sushimasters.com/history-pioneers.htm. 
podrán ser juzgadas, ya que es una forma de adaptación a las nuevas tierras, igual que en los casos del lenguaje, la migración y otros fenómenos sociales. Asimismo se podrá decir que Japón, en cuanto al sushi, está en una etapa de internacionalización con la aplicación de ingredientes no clásicos como carne. Con todo esto, se deduce que el sushi está al menos en vías de enriquecimiento y globalización alimenticia.

\section{Referencias bibliográficas}

Hosking, Richard, Diccionario de la cocina japonesa: Ingredientes y cultura, Zendrera Zariquiey, Barcelona, 2001.

http://www.sushihome.cl/historia.htm.

Kijima, Naomi, Bento Boxes: Japanese Meals on the Go, Graph-sha, Tokio, 2002.

Kodansha International Ltd., Todo sobre Japón, Kodansha International Ltd., Tokio, 2003.

Kodansha Ltd., Kodansha Encyclopedia of Japan, núm. 7, Kodansha, Tokio, 1983.

Kumakura, Isao, "The Globalization of Japanese Food", Food Culture, vol. 1, núm. 200, pp. 5-7.

Morieda, Takashi, Sushi • sushi • sushi, PHP Interface, Tokio, 2002.

Rodger, Jacky (ed.), The Sushi Menu, Cross Media Ltd., Londres, 1998.

Shinmura, Izuru (ed.), Kojien, Iwanami Shoten, Tokio, 1978.

Wai-ming, y N. G. Benjamín, "Imaging and Consuming Japanese Food in Hong Kong, SAR, China: A Study of Culinary Domestication and Hybridization", Asian Profile, vol. 34, núm. 4, 2006. 